FedUni ResearchOnline

http://researchonline.federation.edu.au

This is the author's accepted version of the following publication:

Kruger, A., Minchenko, L., Outrata, J. (2014) On relaxing the

Mangasarian-Fromovitz constraint qualification. Positivity, 18(1),

171-189.

The version displayed here may differ from the final published version.

The final publication is available at:

http://doi.org/10.1007/s11117-013-0238-4

Copyright (C) 2013 Springer Basel 


\title{
On Relaxing the Mangasarian-Fromovitz Constraint Qualification
}

\author{
Alexander Y. Kruger • Leonid \\ Minchenko • Jiří V. Outrata
}

Received: date / Accepted: date

\begin{abstract}
For the classical nonlinear program, two new relaxations of the Mangasarian-Fromovitz constraint qualification are discussed and their relationship with some standard constraint qualifications is examined. In particular, we establish the equivalence of one of these constraint qualifications with the recently suggested by Andreani et al. Constant rank of the subspace component constraint qualification. As an application, we make use of this new constraint qualification in the local analysis of the solution map to a parameterized equilibrium problem, modeled by a generalized equation.
\end{abstract}

Keywords nonlinear programming $\cdot$ regularity conditions $\cdot$ constraint qualifications · Lagrange multipliers · Mangasarian-Fromovitz constraint qualification · Constant rank constraint qualification

Mathematics Subject Classification (2000) 49K40 $90 \mathrm{C} 30 \cdot 90 \mathrm{C} 48$

\footnotetext{
A. Kruger $(\bowtie)$

Centre for Informatics and Applied Optimization, School of Science, Information Technology and Engineering, University of Ballarat, POB 663, Ballarat, Vic, 3350, Australia E-mail: a.kruger@ballarat.edu.au

L. Minchenko

Department of Informatics, Belorussian State University of Informatics and Radioelectronics, 220027 Minsk, Belarus

E-mail: leonidm@insoftgroup.com

J. Outrata

Institute of Information Theory and Automation, Academy of Sciences of the Czech Republic, 18208 Prague, Czech Republic

E-mail: outrata@utia.cas.cz
} 


\section{Introduction}

In this article, we examine regularity properties of the following constraint system:

$$
C=\left\{x \in \mathbb{R}^{n} \mid f_{i}(x)=0, i \in I_{1} ; \quad f_{i}(x) \leq 0, i \in I_{2}\right\},
$$

where $I_{1}$ and $I_{2}$ are finite index sets: $I_{1}=\{1, \ldots, l\}$ and $I_{2}=\{l+1, \ldots, m\}$; $l$ and $m$ are nonnegative integers, $0 \leq l \leq m$. If either $l=0$ or $l=m$, then, respectively, either $I_{1}$ or $I_{2}$ is empty.

System (1) can represent, e.g., the set of admissible points (feasible set) in the general nonlinear programming problem:

$$
\text { Minimize } f_{0}(x) \text { subject to } x \in C \text {. }
$$

The functions $f_{i}: \mathbb{R}^{n} \rightarrow \mathbb{R}, i=0, \ldots, m$, are assumed continuously differentiable near some $\bar{x} \in C$.

The Lagrange function for problem (1)-(2) is defined in the usual way:

$$
L(x, \lambda):=f_{0}(x)+\sum_{i=1}^{m} \lambda_{i} f_{i}(x), \quad x \in \mathbb{R}^{n}, \lambda=\left(\lambda_{1}, \ldots \lambda_{m}\right) \in \mathbb{R}^{m} .
$$

Given an $x \in C$, one can define the corresponding set of Lagrange multipliers: $\Lambda(x):=\left\{\lambda=\left(\lambda_{1}, \ldots \lambda_{m}\right) \in \mathbb{R}^{m} \mid \nabla_{x} L(x, \lambda)=0, \quad \lambda_{i} \geq 0, \lambda_{i} f_{i}(x)=0, i \in I_{2}\right\}$.

The main set of necessary optimality conditions for problem (1)-(2) Karush-Kuhn-Tucker (KKT) conditions - consist in the existence of Lagrange multipliers: if $\bar{x}$ is a local minimizer in problem $(1)-(2)$, then $\Lambda(\bar{x}) \neq \emptyset$, provided certain regularity conditions, usually referred to as constraint qualifications (CQ), are satisfied. The most well known and widely used one is the Mangasarian-Fromovitz constraint qualification (MFCQ) [21].

Given an $x \in C$, it is typical to define the subset

$$
I_{2}(x):=\left\{i \in I_{2} \mid f_{i}(x)=0\right\}
$$

of active (at $x$ ) inequality constraints' indices.

Definition 1 MFCQ is satisfied at $\bar{x} \in C$ if

(i) the vectors $\nabla f_{i}(\bar{x}), i \in I_{1}$, are linearly independent;

(ii) there exists a $z \in \mathbb{R}^{n}$ such that

$$
\left\langle\nabla f_{i}(\bar{x}), z\right\rangle=0, i \in I_{1}, \quad\left\langle\nabla f_{i}(\bar{x}), z\right\rangle<0, i \in I_{2}(\bar{x}) .
$$

Unfortunately, MFCQ fails for many important problems like, e.g., mathematical programs with complementarity constraints [30].

A much weaker constraint qualification still guaranteeing the fulfillment of the KKT conditions at a local minimizer is credited to Abadie [1] (see, 
e.g., $\underline{[5,} \underline{6]}$ ). Let $\bar{x} \in C$. Recall the definition of the tangent (also known as Bouligand or contingent) cone $\underline{[5]}$ to $C$ at $\bar{x}$ :

$$
\begin{aligned}
T_{C}(\bar{x}) & :=\operatorname{Limsup}_{\alpha \rightarrow+\infty} \alpha(C-\bar{x}) \\
& :=\left\{z \in \mathbb{R}^{n} \mid \exists\left\{x_{k}\right\} \subset C, \exists\left\{\alpha_{k}\right\} \rightarrow+\infty \text { such that } \alpha_{k}\left(x_{k}-\bar{x}\right) \rightarrow z\right\} .
\end{aligned}
$$

This is a general definition applicable to any set $C$. If this set is given by smooth equalities and inequalities (1), one can consider the linearized cone to $C$ at $\bar{x}$ :

$$
\Gamma_{C}(\bar{x}):=\left\{z \in \mathbb{R}^{n} \mid\left\langle\nabla f_{i}(\bar{x}), z\right\rangle=0, i \in I_{1}, \quad\left\langle\nabla f_{i}(\bar{x}), z\right\rangle \leq 0, i \in I_{2}(\bar{x})\right\} .
$$

Definition 2 The Abadie constraint qualification (ACQ) is satisfied at $\bar{x} \in C$ if

$$
T_{C}(\bar{x})=\Gamma_{C}(\bar{x}) .
$$

ACQ can be weakened further if the cones $T_{C}(\bar{x})$ and $\Gamma_{C}(\bar{x})$ in $(4)$ are replaced by their polar cones. This condition is known as Guignard constraint qualification [10].

The main drawback of the Abadie and Guignard CQs is that they are difficult to verify.

Several other CQs are known within the range between MFCQ and ACQ, like the Constant positive linear dependence condition $[4,33]$ and the series of its relaxations due to Andreani et al.: the Relaxed constant positive linear dependence condition [2], the Constant rank of the subspace component (CRSC) condition [3] and the Constant positive generator condition [3] as well as the Constant rank Mangasarian-Fromovitz constraint qualification (CRMFCQ) and the Relaxed Mangasarian-Fromovitz constraint qualification (RMFCQ) defined in [23].

The last two conditions will be discussed in more detail in the next section. Particularly, we are going to show that conditions CRSC and RMFCQ are equivalent.

There exist also conditions which are independent of MFCQ, like Constant rank constraint qualification introduced by Janin $\underline{[16]}$ and later studied by many authors (see, e.g., [19]).

Definition 3 The Constant rank constraint qualification (CRCQ) is satisfied at $\bar{x} \in C$ if there exists a neighbourhood $V(\bar{x})$ of $\bar{x}$ such that, for any index set $J \subset I_{1} \cup I_{2}(\bar{x})$, the system of vectors $\left\{\nabla f_{i}(x), i \in J\right\}$ has constant rank in $V(\bar{x})$.

The last condition is also difficult to verify. Besides, it can be too restrictive in many important situations. A relaxation of CRCQ was introduced in $[22,24]$.

Definition 4 The Relaxed constant rank constraint qualification (RCRCQ) is satisfied at $\bar{x} \in C$ if there exists a neighbourhood $V(\bar{x})$ of $\bar{x}$ such that, for any index set $J \subset I_{2}(\bar{x})$, the system of vectors $\left\{\nabla f_{i}(x), i \in I_{1} \cup J\right\}$ has constant rank in $V(\bar{x})$. 
Some examples of application of RCRCQ can be found in [24]. However, when $I_{2}(\bar{x})$ is large, verifying this condition can still be a challenging job. Similar to CRCQ, condition RCRCQ is independent of MFCQ and implies RMFCQ.

For the relationships among various CQs we refer the reader to [5, Chapter 5]; see also [3]. The relationships between MFCQ, CRCQ and RCRCQ and some applications of these conditions are presented in $[19,20]$.

The question of validity of KKT conditions at local minimizers is closely connected with stability properties of canonically perturbed constraint systems which, a fortiori, play an important role in generalized differential calculus, cf., e.g., $[11,19]$. It follows that some qualification conditions are needed also in problems of second order analysis when one analyzes, for instance, solution maps to parameterized generalized equations or, in particular, parameterized KKT systems $[14,28,34]$. Also the notion of amenable set [38, Definition 10.23], very useful in second order analysis, relies on (a generalized version of) MFCQ. It seems, however, that even in this area the standardly used CQs could be replaced by suitable relaxations. In Section 5 we attempt to develop this idea on the basis of RMFCQ.

The structure of the paper is as follows. In the next section, we discuss two successive relaxations of MFCQ, the second one being also a relaxation of CRCQ while still implying ACQ. Its relationship with (in fact, equivalence to) CRSC is also discussed. Well-posedness and robustness properties of CQs (particularly, CRMFCQ and RMFCQ) are discussed in Section 3. In Section 4, we show that RMFCQ implies the error bound property under the assumption that the gradients of the functions involved in (1) are locally Lipschitz continuous. Section 5 is devoted to an application of RMFCQ in second order analysis.

\section{Relaxed Mangasarian-Fromovitz Constraint Qualifications}

The most straightforward way of relaxing MFCQ is to replace the linear independence condition in Definition 1 by the constant rank one.

Definition 5 The Constant rank Mangasarian-Fromovitz constraint qualification (CRMFCQ) is satisfied at $\bar{x} \in C$ if

(i) the system of vectors $\left\{\nabla f_{i}(x), i \in I_{1}\right\}$ has constant rank in a neighbourhood of $\bar{x}$;

(ii) there exists a $z \in \mathbb{R}^{n}$ such that

$$
\left\langle\nabla f_{i}(\bar{x}), z\right\rangle=0, i \in I_{1}, \quad\left\langle\nabla f_{i}(\bar{x}), z\right\rangle<0, i \in I_{2}(\bar{x}) .
$$

Definition 5 was introduced in [23] where the term Extended MangasarianFromovitz condition was used. 
For further relaxation of MFCQ, one needs to have a closer look at the structure of the set of active indices $I_{2}(\bar{x})$. Denote

$$
\begin{aligned}
I_{2}^{0}(\bar{x}) & :=\left\{i \in I_{2}(\bar{x}) \mid\left\langle\nabla f_{i}(\bar{x}), z\right\rangle=0 \text { for all } z \in \Gamma_{C}(\bar{x})\right\} \\
I_{2}^{-}(\bar{x}) & :=\left\{i \in I_{2}(\bar{x}) \mid\left\langle\nabla f_{i}(\bar{x}), z\right\rangle<0 \text { for some } z \in \Gamma_{C}(\bar{x})\right\} .
\end{aligned}
$$

Obviously $I_{2}^{0}(\bar{x}) \cap I_{2}^{-}(\bar{x})=\emptyset$ and $I_{2}(\bar{x})=I_{2}^{0}(\bar{x}) \cup I_{2}^{-}(\bar{x})$.

The next property was also introduced in [23] under the name Generalized Mangasarian-Fromovitz condition.

Definition 6 The Relaxed Mangasarian-Fromovitz constraint qualification (RMFCQ) is satisfied at $\bar{x} \in C$ if

(i) the system of vectors $\left\{\nabla f_{i}(x), i \in I_{1} \cup I_{2}^{0}(\bar{x})\right\}$ has constant rank in a neighbourhood of $\bar{x}$;

(ii) there exists a $z \in \mathbb{R}^{n}$ such that

$$
\left\langle\nabla f_{i}(\bar{x}), z\right\rangle=0, i \in I_{1} \cup I_{2}^{0}(\bar{x}), \quad\left\langle\nabla f_{i}(\bar{x}), z\right\rangle<0, i \in I_{2}^{-}(\bar{x}) .
$$

The second condition in the above definition is always satisfied, thanks to the definitions of the sets $I_{2}^{0}(\bar{x})$ and $I_{2}^{-}(\bar{x})$, and can be dropped. Indeed, if $I_{2}^{-}(\bar{x})=\emptyset$, the condition holds trivially. If $i \in I_{2}^{-}(\bar{x})$, then, for any $z \in \Gamma_{C}(\bar{x})$, it holds $\left\langle\nabla f_{i}(\bar{x}), z\right\rangle \leq 0$ and there exists a $z_{i} \in \Gamma_{C}(\bar{x})$ such that $\left\langle\nabla f_{i}(\bar{x}), z_{i}\right\rangle<$ 0 . Set $z^{\circ}:=\sum_{i \in I_{2}^{-}(\bar{x})} z_{i}$. Then $z^{\circ} \in \Gamma_{C}(\bar{x})$ and consequently $\left\langle\nabla f_{i}(\bar{x}), z^{\circ}\right\rangle=0$ for $i \in I_{1} \cup I_{2}^{0}(\bar{x})$. At the same time, for $i \in I_{2}^{-}(\bar{x})$, we have

$$
\left\langle\nabla f_{i}(\bar{x}), z^{\circ}\right\rangle=\sum_{j \in I_{2}^{-}(\bar{x})}\left\langle\nabla f_{i}(\bar{x}), z_{j}\right\rangle=\sum_{j \in I_{2}^{-}(\bar{x}) \backslash\{i\}}\left\langle\nabla f_{i}(\bar{x}), z_{j}\right\rangle+\left\langle\nabla f_{i}(\bar{x}), z_{i}\right\rangle<0 .
$$

In the rest of the paper we use the following shortened version of Definition 6 .

Definition $6^{\prime}$ The Relaxed Mangasarian-Fromovitz constraint qualification (RMFCQ) is satisfied at $\bar{x} \in C$ if the system of vectors $\left\{\nabla f_{i}(x), i \in\right.$ $\left.I_{1} \cup I_{2}^{0}(\bar{x})\right\}$ has constant rank in a neighbourhood of $\bar{x}$.

All implications in the following diagram, except the last one, are straightforward. The last implication is justified by Theorem 1 below.

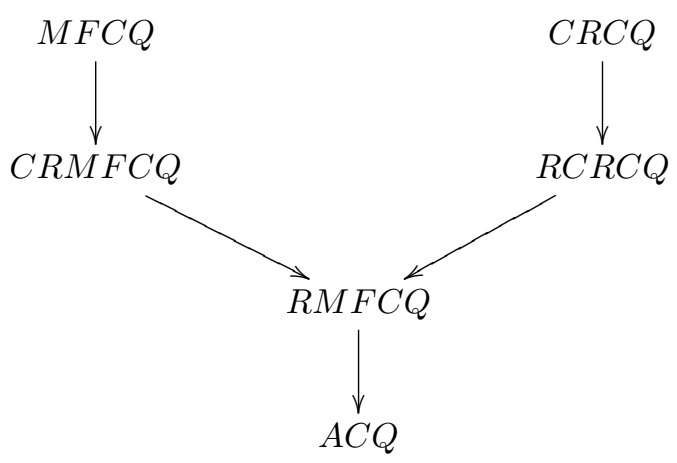


The next theorem shows that RMFCQ, being weaker than both MFCQ and CRMFCQ, is still stronger than ACQ and, hence, sufficient to guarantee the validity of the KKT conditions for problem (1)-(2).

Theorem 1 If RMFCQ is satisfied at $\bar{x} \in C$, then $T_{C}(\bar{x})=\Gamma_{C}(\bar{x})$.

Proof The inclusion $T_{C}(\bar{x}) \subset \Gamma_{C}(\bar{x})$ is always true by the definition of the tangent cone. We only need to prove the opposite inclusion.

Let RMFCQ be satisfied at $\bar{x} \in C$. Then

$$
\begin{aligned}
\operatorname{ri} \Gamma_{C}(\bar{x})=\left\{z \in \mathbb{R}^{n} \mid\left\langle\nabla f_{i}(\bar{x}), z\right\rangle\right. & =0, i \in I_{1} \cup I_{2}^{0}(\bar{x}), \\
\left\langle\nabla f_{i}(\bar{x}), z\right\rangle & \left.<0, i \in I_{2}^{-}(\bar{x})\right\} .
\end{aligned}
$$

Indeed, if $I_{2}^{-}(\bar{x})=\emptyset$, the equality is trivial. Suppose $I_{2}^{-}(\bar{x}) \neq \emptyset$. The following representation for the affine hull of $\Gamma_{C}(\bar{x})$ is straightforward:

$$
\text { aff } \Gamma_{C}(\bar{x})=\left\{z \in \mathbb{R}^{n} \mid\left\langle\nabla f_{i}(\bar{x}), z\right\rangle=0, i \in I_{1} \cup I_{2}^{0}(\bar{x})\right\} .
$$

Define a convex (sublinear) function $h: \mathbb{R}^{n} \rightarrow \mathbb{R}_{\infty}:=\mathbb{R} \cup\{+\infty\}$ :

$$
h(z):= \begin{cases}\max _{i \in I_{2}^{-}(\bar{x})}\left\langle\nabla f_{i}(\bar{x}), z\right\rangle, & z \in \operatorname{aff} \Gamma_{C}(\bar{x}), \\ +\infty, & \text { otherwise. }\end{cases}
$$

Then $\Gamma_{C}(\bar{x})=\left\{z \in \mathbb{R}^{n} \mid h(z) \leq 0\right\}$ and there exists a $z \in \mathbb{R}^{n}$ such that $h(z)<0$. Hence, by [37, Corollary 7.6.1],

$$
\operatorname{ri} \Gamma_{C}(\bar{x})=\left\{z \in \mathbb{R}^{n} \mid h(z)<0\right\},
$$

which implies (7).

Next we are going to show that ri $\Gamma_{C}(\bar{x}) \subset T_{C}(\bar{x})$. Let $z \in \operatorname{ri} \Gamma_{C}(\bar{x})$. Then for any sequences $\left\{x_{k}\right\} \subset \mathbb{R}^{n}$ and $\left\{\alpha_{k}\right\} \subset \mathbb{R}$ such that $\alpha_{k} \rightarrow \infty$ and $\alpha_{k}\left(x_{k}-\right.$ $\bar{x}) \rightarrow z$ as $k \rightarrow \infty$, it holds $f_{i}\left(x_{k}\right)<0$ for all $i \in I_{2} \backslash I_{2}^{0}(\bar{x})=\left(I_{2} \backslash I_{2}(\bar{x})\right) \cup I_{2}^{-}(\bar{x})$ and all sufficiently large $k$. Indeed, we obviously have $x_{k} \rightarrow \bar{x}$ as $k \rightarrow \infty$, and consequently, for all sufficiently large $k$, it holds $f_{i}\left(x_{k}\right)<0$ for all $i \in I_{2} \backslash I_{2}(\bar{x})$. If $i \in I_{2}^{-}(\bar{x})$, then $\left\langle\nabla f_{i}(\bar{x}), z\right\rangle<0$ and denoting $z_{k}:=\alpha_{k}\left(x_{k}-\bar{x}\right)$, we have

$$
\begin{aligned}
f_{i}\left(x_{k}\right) & =f_{i}(\bar{x})+\left\langle\nabla f_{i}(\bar{x}), x_{k}-\bar{x}\right\rangle+o\left(\alpha_{k}^{-1}\right) \\
& =\alpha_{k}^{-1}\left(\left\langle\nabla f_{i}(\bar{x}), z_{k}\right\rangle+\alpha_{k} o\left(\alpha_{k}^{-1}\right)\right)<0
\end{aligned}
$$

for all sufficiently large $k$.

Let $r>0$ denote the rank of the system of vectors $\left\{\nabla f_{i}(\bar{x}), i \in I_{1} \cup I_{2}^{0}(\bar{x})\right\}$. Due to Definition $6^{\prime}$, it remains the same if we consider instead the system of vectors $\left\{\nabla f_{i}(x), i \in I_{1} \cup I_{2}^{0}(\bar{x})\right\}$ for $x$ in a neighbourhood of $\bar{x}$. We can assume without loss of generality that $I_{1} \cup I_{2}^{0}(\bar{x})=\{i \in \mathbb{N} \mid 1 \leq i \leq r+q\}$ for some integer $q \geq 0$, and the vectors $\left\{\nabla f_{i}(\bar{x}), i=1, \ldots, r\right\}$ are linearly independent. Then, using the inverse function theorem, it is not difficult to establish (cf. [4, Lemma 3.2]) the existence of continuously differentiable functions $\phi_{i}: \mathbb{R}^{r} \rightarrow \mathbb{R}$, 
$i=1, \ldots, q$, such that $f_{r+i}(x)=\phi_{i}\left(f_{1}(x), \ldots, f_{r}(x)\right), i=1, \ldots, q$, for all $x$ near $\bar{x}$. Since $\bar{x} \in C$, it follows immediately that $\phi_{i}\left(0_{\mathbb{R}^{r}}\right)=0, i=1, \ldots, q$.

Now consider the system of equations

$$
f_{i}(\bar{x}+t z+x)=0, \quad i=1,2, \ldots, r,
$$

with respect to $t \in \mathbb{R}$ and $x \in \mathbb{R}^{n}$. Obviously $(0,0) \in \mathbb{R} \times \mathbb{R}^{n}$ is a solution. The system has full rank $r \leq n$. Let $x=\left(x_{1}, \ldots, x_{n}\right)$ and suppose without loss of generality that the above system is of rank $r$ with respect to the first $r$ components of $x$. Denote $u=\left(x_{1}, \ldots, x_{r}\right) \in \mathbb{R}^{r}$ and $v=\left(x_{r+1}, \ldots, x_{n}\right) \in$ $\mathbb{R}^{n-r}$. Then $x=(u, v)$ with the convention that $x=u$ if $r=n$. By the implicit function theorem (see, e.g., [8]), system (8) defines in a neighbourhood of $(0,0,0) \in \mathbb{R} \times \mathbb{R}^{r} \times \mathbb{R}^{n-r}$ a continuously differentiable function $(t, v) \rightarrow u(t, v)$ such that $f_{i}(\bar{x}+t z+(u(t, v), v))=0$ for all $i=1,2, \ldots, r$ and all $(t, v)$ near $(0,0) \in \mathbb{R} \times \mathbb{R}^{n}, u(0,0)=0$, and $\partial u(0,0) / \partial t=0$. Then $x:=x(t):=(u(t, 0), 0)$ satisfies system (8) for all $t$ near 0 and $x(t) / t \rightarrow 0$ as $t \downarrow 0$. Besides, for all $t$ near 0 , one has

$$
f_{i}(\bar{x}+t z+x(t))=\phi_{i-r}\left(0_{\mathbb{R}^{r}}\right)=0, \quad i=r+1, \ldots, r+q .
$$

Taking $\alpha_{k}:=k$ and $x_{k}:=\bar{x}+k^{-1} z+x\left(k^{-1}\right), k=1,2, \ldots$, we see that

$$
\alpha_{k}\left(x_{k}-\bar{x}\right)=z+k x\left(k^{-1}\right) \rightarrow z
$$

as $k \rightarrow \infty$ and

$$
f_{i}\left(x_{k}\right)=0, i \in I_{1} \cup I_{2}^{0}(\bar{x}), \quad f_{i}\left(x_{k}\right)<0, i \in I_{2} \backslash I_{2}^{0}(\bar{x}),
$$

that is, $x_{k} \in C$ for all sufficiently large $k$. Hence, $z \in T_{C}(\bar{x})$, and consequently ri $\Gamma_{C}(\bar{x}) \subset T_{C}(\bar{x})$ which implies $\Gamma_{C}(\bar{x}) \subset T_{C}(\bar{x})$ by [37, Theorem 6.3].

Condition RMFCQ can be strictly weaker than MFCQ.

\section{Example 1 Let}

$$
\begin{aligned}
C:=\left\{x=\left(x_{1}, x_{2}\right) \in \mathbb{R}^{2} \mid\right. & -x_{1}^{2}+x_{2} \leq 0,-x_{1}^{2}-x_{2} \leq 0, \\
& \left.-x_{1}+x_{2} \leq 0, x_{1}-x_{2} \leq 0, x_{1}-1 \leq 0\right\} .
\end{aligned}
$$

It is easy to check that $\bar{x}=(0,0)$ is an isolated point of $C$. Define

$$
\begin{gathered}
f_{1}(x):=-x_{1}^{2}+x_{2}, f_{2}(x):=-x_{1}^{2}-x_{2}, \\
f_{3}(x):=-x_{1}+x_{2}, f_{4}(x):=x_{1}-x_{2}, f_{5}(x):=x_{1}-1 .
\end{gathered}
$$

Then $I_{1}=\emptyset, I_{2}=\{1,2,3,4,5\}, I_{2}(\bar{x})=\{1,2,3,4\}$,

$\nabla f_{1}(x)=\left(\begin{array}{c}-2 x_{1} \\ 1\end{array}\right), \nabla f_{2}(x)=\left(\begin{array}{c}-2 x_{1} \\ -1\end{array}\right), \nabla f_{3}(x)=\left(\begin{array}{c}-1 \\ 1\end{array}\right), \nabla f_{4}(x)=\left(\begin{array}{c}1 \\ -1\end{array}\right)$,

$\Gamma_{C}(\bar{x})=\{(0,0)\}, I_{2}^{0}(\bar{x})=I_{2}(\bar{x}), I_{2}^{-}(\bar{x})=\emptyset$, the system of vectors $\left\{\nabla f_{i}(x), i \in\right.$ $\left.I_{2}^{0}(\bar{x})\right\}$ has rank 2 in a neighbourhood of $\bar{x}$ and of course $\left\langle\nabla f_{i}(\bar{x}), 0_{\mathbb{R}^{2}}\right\rangle=0$, $i \in I_{2}^{0}(\bar{x})$. Thus RMFCQ is satisfied at $\bar{x}$. On the other hand, MFCQ is not satisfied at $\bar{x}$ : there is no $z \in \mathbb{R}^{2}$ such that $\left\langle\nabla f_{i}(\bar{x}), z\right\rangle\left\langle 0, i \in I_{2}(\bar{x})\right.$.

CRCQ [16], RCRCQ [24,25], CPLD [4,33], and RCPLD [2] are not satisfied in this example either. 
A new CQ introduced recently by Andreani et al. [3] uses the following set of indices:

$$
I_{2}^{*}(\bar{x}):=\left\{i \in I_{2}(\bar{x}) \mid-\nabla f_{i}(\bar{x}) \in\left(\Gamma_{C}(\bar{x})\right)^{\circ}\right\},
$$

where $\left(\Gamma_{C}(\bar{x})\right)^{\circ}$ denotes the (negative) polar cone to $\Gamma_{C}(\bar{x})$.

Definition 7 ( [3]) The Constant rank of the subspace component (CRSC) condition is satisfied at $\bar{x} \in C$ if the system of vectors $\left\{\nabla f_{i}(x), i \in I_{1} \cup I_{2}^{*}(\bar{x})\right\}$ has constant rank in a neighbourhood of $\bar{x}$.

Observe that $\left\langle\nabla f_{i}(\bar{x}), z\right\rangle \leq 0$ for any $z \in \Gamma_{C}(\bar{x})$ and $i \in I_{2}(\bar{x})$ by definition of $\Gamma_{C}(\bar{x})$. Hence, for any $i \in I_{2}(\bar{x})$, inclusion $-\nabla f_{i}(\bar{x}) \in\left(\Gamma_{C}(\bar{x})\right)^{\circ}$ is equivalent to the equality $\left\langle\nabla f_{i}(\bar{x}), z\right\rangle=0$ being valid for all $z \in \Gamma_{C}(\bar{x})$, that is, $i \in$ $I_{2}^{0}(\bar{x})$. Thus $I_{2}^{*}(\bar{x})=I_{2}^{0}(\bar{x})$, and consequently condition CRSC coincides with RMFCQ.

Since $\left(\Gamma_{C}(\bar{x})\right)^{\circ}$ admits the following representation:

$$
\left(\Gamma_{C}(\bar{x})\right)^{\circ}=\left\{\sum_{i \in I_{1} \cup I_{2}(\bar{x})} \alpha_{i} \nabla f_{i}(\bar{x}) \mid \alpha_{i} \geq 0, i \in I_{2}(\bar{x})\right\},
$$

formula (9) can be slightly simplified:

$$
\begin{aligned}
& I_{2}^{0}(\bar{x})=I_{2}^{*}(\bar{x}) \\
& =\left\{j \in I_{2}(\bar{x}) \mid-\nabla f_{j}(\bar{x}) \in\left\{\sum_{i \in I_{1} \cup I_{2}(\bar{x}) \backslash\{j\}} \alpha_{i} \nabla f_{i}(\bar{x}) \mid \alpha_{i} \geq 0, i \in I_{2}(\bar{x})\right\}\right\} .
\end{aligned}
$$

It is possible to show that RMFCQ is a particular case of a more general CQ due to Penot [32].

Definition 8 The Penot constraint qualification is satisfied at $\bar{x} \in C$ if for any $\bar{z} \in \Gamma_{C}(\bar{x})$ there exists a $z \in \mathbb{R}^{n}$ and a subset $J_{2} \subset I_{2}(\bar{x})$ such that, with $J=I_{1} \cup J_{2}$,

(i) $\left\langle\nabla f_{i}(\bar{x}), \bar{z}\right\rangle=0, i \in J$;

(ii) $\left\langle\nabla f_{i}(\bar{x}), z\right\rangle=0, i \in J, \quad\left\langle\nabla f_{i}(\bar{x}), z\right\rangle<0, i \in I_{2}(\bar{x}) \backslash J_{2}$;

(iii) $T_{C_{0}}(\bar{x})=\left\{y \in \mathbb{R}^{n} \mid\left\langle\nabla f_{i}(\bar{x}), y\right\rangle=0, i \in J\right\}$ where

$C_{0}:=\left\{y \in \mathbb{R}^{n} \mid f_{i}(\bar{x})=0, i \in J\right\}$.

Unfortunately, the Penot constraint qualification is difficult to verify.

\section{Well-posedness and Robustness}

From the point of view of applications, it is important to have regularity/qualification conditions possessing certain robustness.

Definition 9 A CQ at $\bar{x} \in C$ is 
(i) well-posed $\underline{[26]}$ if, once it is satisfied at $\bar{x}$, it is also satisfied at any $x \in C$ near $\bar{x}$.

(ii) robust if, once it is satisfied at $\bar{x}$, it implies that $\Lambda(x) \neq \emptyset$ for any objective function $f_{0}$ and any local minimizer $x$ of problem (1)-(2) in a neigbourhood of $\bar{x}$.

MFCQ, the Constant positive linear dependence condition, CRCQ and RCRCQ, as well as the general quasi-normality condition from [31] are wellposed. At the same time, the Abadie and Guignard CQs are neither well-posed nor robust.

Example 2 Let

$$
C:=\left\{x=\left(x_{1}, x_{2}, x_{3}\right) \in \mathbb{R}^{3} \mid-x_{1}^{3}-x_{2} \leq 0,-x_{1}^{3}+x_{2} \leq 0,-x_{1}-x_{3}^{2} \leq 0\right\} .
$$

Obviously, $\bar{x}=(0,0,0) \in C$. Define

$$
f_{1}(x):=-x_{1}^{3}-x_{2}, f_{2}(x):=-x_{1}^{3}+x_{2}, f_{3}(x):=-x_{1}-x_{3}^{2} .
$$

Then $I_{1}=\emptyset, I_{2}=I_{2}(\bar{x})=\{1,2,3\}$, and ACQ is satisfied at $\bar{x}$ :

$$
T_{C}(\bar{x})=\Gamma_{C}(\bar{x})=\left\{z=\left(z_{1}, z_{2}, z_{3}\right) \in \mathbb{R}^{3} \mid z_{1} \geq 0, z_{2}=0\right\} .
$$

For any $\varepsilon>0$, one can take $x_{\varepsilon}=(0,0, \varepsilon) \in \mathbb{R}^{3}$ which obviously belongs to $C$. The tangent cone remains the same: $T_{C}\left(x_{\varepsilon}\right)=T_{C}(\bar{x})$. However, $I_{2}\left(x_{\varepsilon}\right)=\{1,2\}$ and $\Gamma_{C}\left(x_{\varepsilon}\right)=\left\{z=\left(z_{1}, z_{2}, z_{3}\right) \in \mathbb{R}^{3} \mid z_{2}=0\right\}$. ACQ is not satisfied at $x_{\varepsilon}$.

Moreover, $x_{\varepsilon}$ is obviously a minimizer of the function $x \mapsto f_{0}(x):=x_{1}$ subject to $x \in C$ while the KKT conditions at $x_{\varepsilon}$ produce the following inconsistent system with respect to $\left(\lambda_{1}, \lambda_{2}, \lambda_{3}\right) \in \mathbb{R}^{3}$ :

$$
\begin{gathered}
\left(\begin{array}{l}
1 \\
0 \\
0
\end{array}\right)+\lambda_{1}\left(\begin{array}{c}
0 \\
-1 \\
0
\end{array}\right)+\lambda_{2}\left(\begin{array}{l}
0 \\
1 \\
0
\end{array}\right)+\lambda_{3}\left(\begin{array}{c}
-1 \\
0 \\
-2 \varepsilon
\end{array}\right)=\left(\begin{array}{l}
0 \\
0 \\
0
\end{array}\right), \\
\lambda_{1} \geq 0, \lambda_{2} \geq 0, \lambda_{3}=0 .
\end{gathered}
$$

Theorem 2 CRMFCQ is robust at any $\bar{x} \in C$.

Proof Let CRMFCQ be satisfied at some $\bar{x} \in C$. Then $I_{2}^{-}(\bar{x})=I_{2}(\bar{x})$. Choose $I_{1}^{0} \subset I_{1}$ such that $\left\{\nabla f_{i}(\bar{x}), i \in I_{1}^{0}\right\}$ is a maximal linear independent subsystem of the system of vectors $\left\{\nabla f_{i}(\bar{x}), i \in I_{1}\right\}$. Then $\left\{\nabla f_{i}(x), i \in I_{1}^{0}\right\}$ is a maximal linear independent subsystem of $\left\{\nabla f_{i}(x), i \in I_{1}\right\}$ for all $x \in \mathbb{R}^{n}$ near $\bar{x}$. Denote by $r$ the rank of the system $\left\{\nabla f_{i}(\bar{x}), i \in I_{1}\right\}$ and assume for simplicity that $I_{1}^{0}=\{i=1,2, \ldots, r\}$. Then, as in the proof of Theorem 1 , there exist continuously differentiable functions $\phi_{i}: \mathbb{R}^{r} \rightarrow \mathbb{R}, i \in I_{1} \backslash I_{1}^{0}$, such that $f_{i}(x)=\phi_{i}\left(f_{1}(x), \ldots, f_{r}(x)\right)$ for all $x$ near $\bar{x}$ and $\phi_{i}\left(0_{\mathbb{R}^{r}}\right)=0$. Hence, there exists a neighbourhood $U$ of $\bar{x}$ such that $C \cap U=C_{0} \cap U$, where

$$
C_{0}=\left\{x \in \mathbb{R}^{n} \mid f_{i}(x)=0, i \in I_{1}^{0} ; \quad f_{i}(x) \leq 0, i \in I_{2}\right\} .
$$


With $C_{0}$ replacing $C$, CRMFCQ becomes the standard MFCQ which is well defined and holds true in a neighbourhood of $\bar{x}$. We will keep denotation $U$ for this possibly smaller neighbourhood. Hence, for any objective function $f_{0}$ and any its local minimizer on $C \cap U$, there exist Lagrange multipliers $\lambda_{i}$, $i \in I_{1}^{0} \cup I_{2}$. Now it is sufficient to define additionally $\lambda_{i}=0, i \in I_{1} \backslash I_{1}^{0}$.

The following important lemma proved in [3, Lemma 5.3] is the key tool in establishing the well-posedness of RMFCQ. It is also used in the proof of Theorem 4 in the next section.

Lemma 1 If RMFCQ is satisfied at $\bar{x}$, then $I_{2}^{0}(x)=I_{2}^{0}(\bar{x})$ for all $x \in C$ near $\bar{x}$.

The next theorem is a direct consequence of Lemma 1.

Theorem 3 RMFCQ is well-posed at any $\bar{x} \in C$.

\section{RMFCQ and Error Bounds}

In this section, we show that RMFCQ implies the error bounds property.

Definition 10 The constraint system $C$ defined by (1) satisfies the error bound property at $\bar{x} \in C$ if there exists an $\alpha>0$ such that

$$
d(x, C) \leq \alpha \max \left\{\left|f_{i}(x)\right|, i \in I_{1} ; \quad f_{i}(x), i \in I_{2}\right\}
$$

for all $x$ near $\bar{x}$

The concept of error bounds in mathematical programming goes back to Robinson [35]. This property is also known as $R$-regularity $[22,25]$.

Theorem 4 Let the gradients $\nabla f_{i}(x), i=1,2, \ldots, m$, be Lipschitz continuous in a neighbourhood of $\bar{x} \in C$. If $R M F C Q$ is satisfied at $\bar{x}$, then $C$ satisfies the error bound property at $\bar{x}$.

Given a $y \in X$, let $\Pi_{C}(y)$ denote its (possibly multivalued) projection on $C$ corresponding to the Euclidean norm $\|\cdot\|$ on $\mathbb{R}^{n}$, i.e., $x \in \Pi_{C}(y)$ if and only if $x$ is a minimizer of the function $u \rightarrow f_{y}(u):=\|u-y\|$ on $C$. $f_{y}$ is differentiable at any $u \neq y$ with $\nabla f_{y}(u) \neq 0$. Assuming that $y \notin C$, denote by $\Lambda_{y}(x)$ the corresponding set of Lagrange multipliers at $x \in \Pi_{C}(y)$ :

$$
\begin{array}{r}
\Lambda_{y}(x):=\left\{\lambda=\left(\lambda_{1}, \ldots \lambda_{m}\right) \in \mathbb{R}^{m} \mid \nabla f_{y}(x)+\sum_{i=1}^{m} \lambda_{i} \nabla f_{i}(x)=0,\right. \\
\left.\lambda_{i} \geq 0, \lambda_{i} f_{i}(x)=0, i \in I_{2}\right\} .
\end{array}
$$

For an $r>0$, denote

$$
\Lambda_{y}^{r}(x):=\Lambda_{y}(x) \cap\left(r \mathbb{B}_{m}\right)
$$

where $\mathbb{B}_{m}$ is the unit ball in $\mathbb{R}^{m}$.

The next lemma which is a direct consequence of [25, Theorem 2] plays a crucial role in the proof of Theorem 4 . 
Lemma 2 Let the gradients $\nabla f_{i}(x), i=1,2, \ldots, m$, be Lipschitz continuous in a neighbourhood of $\bar{x} \in \operatorname{bd} C$ (the boundary of $C$ ) and there exists an $r>0$ such that $\Lambda_{y}^{r}(x) \neq \emptyset$ for any $y \notin C$ in a neighbourhood of $\bar{x}$ and any $x \in \Pi_{C}(y)$. Then $C$ satisfies the error bound property at $\bar{x}$.

Proof (of Theorem 4) If $\bar{x}$ lies in the interior of $C$, the error bound property holds trivially. Suppose $\bar{x} \in \operatorname{bd} C$, RMFCQ is satisfied at $\bar{x}$ while the error bound property does not hold at $\bar{x}$. By Lemma 2, there exist sequences $\left\{y_{k}\right\}$ and $\left\{x_{k}\right\}$ such that $y_{k} \notin C, x_{k} \in \Pi_{C}\left(y_{k}\right)(k=1,2, \ldots), y_{k} \rightarrow \bar{x}$, and

$$
d\left(0, \Lambda_{y_{k}}\left(x_{k}\right)\right) \rightarrow \infty \quad \text { as } \quad k \rightarrow \infty .
$$

We obviously have $x_{k} \rightarrow \bar{x}$. Passing to a subsequence if necessary, we can suppose that $I_{2}\left(x_{k}\right)=I_{2}^{*}\left(\subset I_{2}(\bar{x})\right)$ is constant and, making use of Theorem 3 , RMFCQ is satisfied at $x_{k}$ for all $k=1,2, \ldots$ Hence, for all $k$,

$$
\Lambda_{y_{k}}\left(x_{k}\right) \neq \emptyset ; \quad f_{i}\left(x_{k}\right)=0, i \in I_{1} \cup I_{2}^{*} ; \quad f_{i}\left(x_{k}\right)<0, i \in I_{2} \backslash I_{2}^{*} .
$$

By Lemma 1, we can also assume that $I_{2}^{0}\left(x_{k}\right)=I_{2}^{0}(\bar{x}) \subset I_{2}^{*}$ for all $k$.

Choose a subset $J \subset I_{1} \cup I_{2}^{0}(\bar{x})$ such that $\left\{\nabla f_{i}(\bar{x}), i \in J\right\}$ is a maximal linear independent subsystem of the system of vectors $\left\{\nabla f_{i}(\bar{x}), i \in I_{1} \cup I_{2}^{0}(\bar{x})\right\}$. Thanks to RMFCQ, we can assume that, for any $k,\left\{\nabla f_{i}\left(x_{k}\right), i \in J\right\}$ is a maximal linearly independent subsystem of the system $\left\{\nabla f_{i}\left(x_{k}\right), i \in I_{1} \cup\right.$ $\left.I_{2}^{0}(\bar{x})\right\}$. Now choose a subset $J^{-} \subset I_{2}^{*} \backslash I_{2}^{0}(\bar{x})$ such that $\left\{\nabla f_{i}\left(x_{k}\right), i \in J \cup\right.$ $\left.J^{-}\right\}$is a maximal linearly independent subsystem of the system of vectors $\left\{\nabla f_{i}\left(x_{k}\right), i \in I_{1} \cup I_{2}^{*}\right\}$. There exists an $i \in I_{1} \cup I_{2}^{*}$ such that $\nabla f_{i}\left(x_{k}\right) \neq 0$, because otherwise, it would follow from definition (10) that $\nabla f_{y_{k}}\left(x_{k}\right)=0$ which is impossible. Hence, $J \cup J^{-} \neq \emptyset$.

There exists a vector $\lambda^{k}=\left(\lambda_{1}^{k}, \ldots \lambda_{m}^{k}\right) \in \Lambda_{y_{k}}\left(x_{k}\right)$ such that

$$
\nabla f_{y}\left(x_{k}\right)+\sum_{i=1}^{m} \lambda_{i}^{k} \nabla f_{i}\left(x_{k}\right)=0 ; \quad \lambda_{i}^{k} \geq 0, i \in I_{2} ; \quad \lambda_{i}^{k}=0, i \notin J \cup J^{-} .
$$

By (11), $\left\|\lambda^{k}\right\| \rightarrow \infty$ as $k \rightarrow \infty$. Without loss of generality, $\lambda_{k} /\left\|\lambda_{k}\right\| \rightarrow \lambda=$ $\left(\lambda_{1}, \ldots, \lambda_{m}\right)$. Then $\|\lambda\|=1$ and it follows from (12) that

$$
\sum_{i=1}^{m} \lambda_{i} \nabla f_{i}(\bar{x})=0 ; \quad \lambda_{i} \geq 0, i \in I_{2} ; \quad \lambda_{i}=0, i \notin J \cup J^{-} .
$$

Since $J^{-} \subset I_{2}^{*} \backslash I_{2}^{0}(\bar{x}) \subset I_{2}(\bar{x}) \backslash I_{2}^{0}(\bar{x})=I_{2}^{-}(\bar{x})$, we have $\lambda_{i}=0$ for all $i \in J^{-}$. Indeed, if $\lambda_{j}>0$ for some $j \in J^{-}$, then, by definitions (5) and (3), there exists a $z \in \mathbb{R}^{n}$ such that

$$
\left\langle\nabla f_{i}(\bar{x}), z\right\rangle=0, i \in I_{1} ; \quad\left\langle\nabla f_{i}(\bar{x}), z\right\rangle \leq 0, i \in I_{2}(\bar{x}) ; \quad\left\langle\nabla f_{j}(\bar{x}), z\right\rangle<0,
$$

and consequently

$$
\sum_{i=1}^{m} \lambda_{i}\left\langle\nabla f_{i}(\bar{x}), z\right\rangle<0
$$


which is impossible in view of (13). Hence, (13) can be rewritten as

$$
\sum_{i \in J} \lambda_{i} \nabla f_{i}(\bar{x})=0 ; \quad \lambda_{i} \geq 0, i \in I_{2},
$$

where not all $\lambda_{i}, i \in J$, are equal zero, but this contradicts the linear independence of the system of vectors $\left\{\nabla f_{i}(\bar{x}), i \in J\right\}$. The proof is completed.

Theorem 4 strengthens [3, Theorem 5.5] which establishes the error bound property under the assumption that the functions $f_{i}, i=1,2, \ldots, m$, are twice differentiable in a neighbourhood of $\bar{x}$.

It was shown in [12, Proposition 1] that the error bound property implies the equality $T_{C}(\bar{x})=\Gamma_{C}(\bar{x})$. Hence, as observed by one of the reviewers, under the assumption of Lipschitz continuity of the gradients $\nabla f_{i}(x), i=1,2, \ldots, m$, Theorem 1 is a consequence of Theorem 4. We do not know if RMFCQ implies the error bound property without this assumption.

\section{An Application in Second Order Analysis}

Recall first a few definitions which will be used in the sequel.

If $x \in C \subset \mathbb{R}^{n}$, then

$$
N_{C}(x):=\left\{x^{*} \in\left(\mathbb{R}^{n}\right)^{*} \mid \limsup _{u \rightarrow x} \frac{\left\langle x^{*}, u-x\right\rangle}{\|u-x\|} \leq 0\right\}
$$

is the Fréchet normal cone to $C$ at $x$. The denotation $u \stackrel{C}{\rightarrow} x$ in the last formula means that $u \rightarrow x$ with $u \in C$. If $x \notin C$, we set $N_{C}(x)=\emptyset$.

If $\bar{x} \in C \subset \mathbb{R}^{n}$, then

$$
\begin{aligned}
\bar{N}_{C}(\bar{x}):=\operatorname{Limsup}_{x \rightarrow \bar{x}} N_{C}(x) & \\
:=\left\{x^{*} \in\left(\mathbb{R}^{n}\right)^{*} \mid\right. & \exists\left\{x_{k}\right\} \subset C, \exists\left\{x_{k}^{*}\right\} \subset\left(\mathbb{R}^{n}\right)^{*} \text { such that } \\
& \left.x_{k} \rightarrow \bar{x}, x_{k}^{*} \rightarrow x^{*}, \text { and } x_{k}^{*} \in N_{C}\left(x_{k}\right)\right\}
\end{aligned}
$$

is the limiting normal cone to $C$ at $\bar{x}$.

If $S\left[\mathbb{R}^{s} \rightrightarrows \mathbb{R}^{n}\right]$ is a multifunction with graph gph $S:=\left\{(p, x) \in \mathbb{R}^{s} \times \mathbb{R}^{n} \mid x \in\right.$ $S(p)\}$ and $(\bar{p}, \bar{x}) \in \operatorname{gph} S$, then the limiting coderivative $\bar{D}^{*} S(\bar{p}, \bar{x})$ of $S$ at $(\bar{p}, \bar{x})$ is defined as

$$
\bar{D}^{*} S(\bar{p}, \bar{x})\left(x^{*}\right):=\left\{p^{*} \in \mathbb{R}^{s} \mid\left(p^{*},-x^{*}\right) \in \bar{N}_{\mathrm{gph} S}(\bar{p}, \bar{x})\right\}, x^{*} \in \mathbb{R}^{n} .
$$

Let us recall some basic stability notions for multifunctions which will be used in the sequel, cf., e.g., $[8,27,38]$. Given a multifunction $S\left[\mathbb{R}^{s} \rightrightarrows \mathbb{R}^{n}\right]$ and a point $(\bar{p}, \bar{x}) \in \operatorname{gph} S$, one has:

(i) $S$ is said to be calm at $(\bar{p}, \bar{x})$ if there are neighborhoods $\mathcal{U}$ of $\bar{p}$ and $\mathcal{V}$ of $\bar{x}$ and a positive scalar $L$ such that

$$
S(p) \cap \mathcal{V} \subset S(\bar{p})+L\|p-\bar{p}\| \mathbb{B}, \quad \forall p \in \mathcal{U} ;
$$


(ii) if, instead of (14), a stronger condition

$$
S\left(p_{1}\right) \cap \mathcal{V} \subset S\left(p_{2}\right)+L\left\|p_{1}-p_{2}\right\| \mathbb{B}, \quad \forall p_{1}, p_{2} \in \mathcal{U}
$$

holds, then $S$ is said to have the Aubin Lipschitz-like property around $(\bar{p}, \bar{x})$;

(iii) if, in addition to (15), for each $p \in \mathcal{U}, S(p) \cap \mathcal{V}$ is a singleton, then $S^{-1}$ is said to be strongly metrically regular at $(\bar{x}, \bar{p})$.

Consider the generalized equation (GE)

$$
0 \in F(p, x)+N_{C}(x)
$$

where $x \in \mathbb{R}^{n}$ is the decision variable, $p \in \mathbb{R}^{s}$ is a parameter, $F\left[\mathbb{R}^{s} \times \mathbb{R}^{n} \rightarrow \mathbb{R}^{n}\right]$ is continuously differentiable, and $C$ is given by (1).

Denote by $S$ the solution map associated with (16), i.e.,

$$
S(p):=\left\{x \in \mathbb{R}^{n} \mid 0 \in F(p, x)+N_{C}(x)\right\} .
$$

Let $(\bar{p}, \bar{x}) \in \operatorname{gph} S$ and let the functions $f_{i}: \mathbb{R}^{n} \rightarrow \mathbb{R}, i=1, \ldots, m$, defining set $C$, be twice continuously differentiable near $\bar{x}$.

In various sensitivity and stability considerations, one usually imposes MFCQ at the reference point $\bar{x}$ to be able to replace (16), locally around $\bar{x}$, by the GE

$$
0 \in F(p, x)+(\nabla f(x))^{T} N_{E}(f(x)),
$$

where $f=\left(f_{1}, \ldots, f_{m}\right)^{T}$ and

$$
E=\left\{0_{\mathbb{R}^{l}}\right\} \times \mathbb{R}_{-}^{m-l} .
$$

Then, by applying appropriate generalized differential calculus rules, one can establish an upper estimate for the limiting coderivative $\bar{D}^{*} S(\bar{p}, \bar{x})$, cf. $\underline{[18,28]}$. In these rules, however, one needs MFCQ again together with a suitable second order CQ, cf. [18, condition (17)] or [28, Theorem 3.1 (ii)]. So, MFCQ at $\bar{x}$ is a key assumption in these developments.

Nevertheless, the possibility of replacing (16) by (17) is available under any well-posed CQ which implies at the same time the calmness at $(0, \bar{x})$ of the perturbation map

$$
\begin{array}{r}
M(v):=\left\{x \in \mathbb{R}^{n} \mid f_{i}(x)+v_{i}=0 \text { for } i \in I_{1},\right. \\
\left.f_{i}(x)+v_{i} \leq 0 \text { for } i \in I_{2}\right\},
\end{array}
$$

where $v=\left(v_{1}, \ldots, v_{m}\right)^{T}$.

Indeed, taking into account that $N_{C}(\bar{x}) \subset \bar{N}_{C}(\bar{x})$, by virtue of the imposed calmness condition one can apply [11, Theorem 4.1] to obtain

$$
N_{C}(\bar{x}) \subset(\nabla f(\bar{x}))^{T} N_{E}(f(\bar{x})) .
$$

Since the opposite inclusion holds true automatically, thanks to the well-posedness, one has

$$
N_{C}(x)=(\nabla f(x))^{T} N_{E}(f(x))
$$


for all $x$ in a neighbourhood of $\bar{x}$. We observe that (20) definitely holds, e.g., under the Relaxed constant rank condition [25, Theorem 1]. It follows from Theorem 4 that RMFCQ can be used as well.

Proposition 1 Suppose RMFCQ holds at $\bar{x}$. Then the perturbation map (19) is calm at $(0, \bar{x})$.

Proof The statement follows immediately from the well known equivalence between the calmness of $(19)$ at $(0, \bar{x})$ and the error bound property (Definition 10) of $C$ at $\bar{x}$ implied by RMFCQ by virtue of Theorem 4 .

By virtue of Theorem 3, we may conclude that, under RMFCQ at $\bar{x}$, equality (20) holds for all $x$ in a neighbourhood of $\bar{x}$. Consequently, (16) can be, locally around $\bar{x}$, replaced by either (17) or the KKT system

$$
\begin{aligned}
& 0=\mathcal{L}(p, x, \lambda), \\
& x \in C, \lambda_{i} \geq 0, \lambda_{i} f_{i}(x)=0, i \in I_{2},
\end{aligned}
$$

where

$$
\mathcal{L}(p, x, \lambda):=F(p, x)+\sum_{i=1}^{m} \lambda_{i} \nabla f_{i}(x)
$$

is the Lagrangian associated with (16).

Define the enhanced solution map $S^{e}\left[\mathbb{R}^{s} \rightrightarrows \mathbb{R}^{n} \times \mathbb{R}^{m}\right]$ by

$$
S^{e}(p):=\left\{(x, \lambda) \in \mathbb{R}^{n} \times \mathbb{R}^{m} \mid \text { system (21) is satisfied }\right\} .
$$

Let $(\bar{p}, \bar{x}, \bar{\lambda}) \in \operatorname{gph} S^{e}$. The limiting coderivative $\bar{D}^{*} S^{e}(\bar{p}, \bar{x}, \bar{\lambda})$ of $S^{e}$ at $(\bar{p}, \bar{x}, \bar{\lambda})$ was computed in $[18]$ in the case when (16) corresponds to stationarity conditions of a parameterized nonlinear program. Unlike [18], we provide now an upper estimate for $\bar{D}^{*} S^{e}(\bar{p}, \bar{x}, \bar{\lambda})$ without requiring MFCQ at $\bar{x}$. In the next statement, we use the polar cone

$$
E^{\circ}=\mathbb{R}^{l} \times \mathbb{R}_{+}^{m-l}
$$

to cone $E$ defined by (18).

Theorem 5 Suppose $R M F C Q$ is fulfilled at $\bar{x}$ and multifunction $\mathcal{M}\left[\mathbb{R}^{n+2 m} \rightrightarrows\right.$ $\mathbb{R}^{s+n+m}$ ] defined by

$$
\mathcal{M}(\xi):=\left\{(p, x, \lambda) \mid \xi \in\left[\begin{array}{c}
\mathcal{L}(p, x, \lambda) \\
-\left(\begin{array}{c}
\lambda \\
f(x)
\end{array}\right)+\operatorname{gph} N_{E^{\circ}}
\end{array}\right]\right\}
$$

is calm at $\left(0_{\mathbb{R}^{n+2 m}}, \bar{p}, \bar{x}, \bar{\lambda}\right)$. Then for any $(a, b) \in \mathbb{R}^{n} \times \mathbb{R}^{m}$ one has

$$
\begin{aligned}
\bar{D}^{*} S^{e}(\bar{p}, \bar{x}, \bar{\lambda})(a, b) \subset\{ & \left(\nabla_{p} F(\bar{p}, \bar{x})\right)^{T} u \mid(u, v) \in \mathbb{R}^{n} \times \mathbb{R}^{m}, \\
& 0=a+\left(\nabla_{x} \mathcal{L}(\bar{p}, \bar{x}, \bar{\lambda})\right)^{T} u+(\nabla f(\bar{x}))^{T} v, \\
& \left.0 \in b+\nabla f(\bar{x}) u+\bar{D}^{*} N_{E^{\circ}}(\bar{\lambda}, f(\bar{x}))(-v)\right\} .
\end{aligned}
$$


Proof Multifunction $\mathcal{M}$ corresponds to the canonical perturbation of the KKT system (21). Denote

$$
\Phi(p, x, \lambda):=\left[\begin{array}{c}
\mathcal{L}(p, x, \lambda) \\
\lambda \\
f(x)
\end{array}\right], \quad G:=\left\{0_{\mathbb{R}^{n}}\right\} \times \operatorname{gph} N_{E^{\circ}},
$$

so that

$$
\operatorname{gph} S^{e}=\{(p, x, \lambda) \mid \Phi(p, x, \lambda) \in G\} .
$$

Thanks to the calmness of multifunction $\mathcal{M}$ at $\left(0_{\mathbb{R}^{n+2 m}}, \bar{p}, \bar{x}, \bar{\lambda}\right)$, we can now invoke [11, Theorem 4.1] to obtain

$$
\bar{N}_{\mathrm{gph} S^{e}}(\bar{p}, \bar{x}, \bar{\lambda}) \subset(\nabla \Phi(\bar{p}, \bar{x}, \bar{\lambda}))^{T} \bar{N}_{G}(\Phi(\bar{p}, \bar{x}, \bar{\lambda})) .
$$

Hence, for all $(a, b) \in \mathbb{R}^{n} \times \mathbb{R}^{m}$ one has

$$
\bar{D}^{*} S^{e}(\bar{p}, \bar{x}, \bar{\lambda})(a, b) \subset\left\{z \in \mathbb{R}^{s} \mid(z,-a,-b)^{T} \in(\nabla \Phi(\bar{p}, \bar{x}, \bar{\lambda}))^{T} \bar{N}_{G}(\Phi(\bar{p}, \bar{x}, \bar{\lambda}))\right\} .
$$

It remains to observe that

$$
\begin{gathered}
\nabla_{p} \Phi(\bar{p}, \bar{x}, \bar{\lambda})=\left[\begin{array}{c}
\nabla_{p} F(\bar{p}, \bar{x}) \\
0 \\
0
\end{array}\right], \quad \nabla_{x} \Phi(\bar{p}, \bar{x}, \bar{\lambda})=\left[\begin{array}{c}
\nabla_{x} \mathcal{L}(\bar{p}, \bar{x}, \bar{\lambda}) \\
0 \\
\nabla f(\bar{x})
\end{array}\right], \\
\nabla_{\lambda} \Phi(\bar{p}, \bar{x}, \bar{\lambda})=\left[\begin{array}{c}
(\nabla f(\bar{x}))^{T} \\
I_{m} \\
0
\end{array}\right],
\end{gathered}
$$

where $I_{m}$ is the identity $m \times m$ matrix, and

$$
\begin{aligned}
\bar{N}_{G}(\Phi(\bar{p}, \bar{x}, \bar{\lambda})) & =\mathbb{R}^{n} \times \bar{N}_{\mathrm{gph} N_{E^{\circ}}}(\bar{\lambda}, f(\bar{x})) \\
& =\left\{(u, w, v) \in \mathbb{R}^{n} \times \mathbb{R}^{m} \times \mathbb{R}^{m} \mid w \in \bar{D}^{*} N_{E^{\circ}}(\bar{\lambda}, f(\bar{x}))(-v)\right\} .
\end{aligned}
$$

Formula (23) follows immediately.

The limiting coderivative $\bar{D}^{*} N_{E^{\circ}}(\bar{\lambda}, f(\bar{x}))$ in (23) can be easily computed directly, cf. [14, proof of Proposition 2]. The verification of the calmness assumption in Theorem 5 seems to be a more challenging job. Various sufficient conditions can be found in the literature, cf., e.g., $[9,15]$. Sometimes one can also use the following statement based on the calmness criterion in [17, Theorem 2.5].

Proposition 2 Suppose that $\nabla_{p} F(\bar{p}, \bar{x})$ is surjective and multifunction

$$
\mathcal{N}(\beta):=\left\{(x, \lambda) \mid \beta+\left(\begin{array}{c}
\lambda \\
f(x)
\end{array}\right) \in \operatorname{gph} N_{E^{\circ}}\right\}
$$

is calm at $\left(0_{\mathbb{R}^{2 m}}, \bar{x}, \bar{\lambda}\right)$. Then multifunction $\mathcal{M}$ given by $(22)$ is calm at $\left(0_{\mathbb{R}^{n+2 m}}, \bar{p}, \bar{x}, \bar{\lambda}\right)$. 
Proof Let $\xi=\left(\xi_{1}, \xi_{2}, \xi_{3}\right) \in \mathbb{R}^{n} \times \mathbb{R}^{m} \times \mathbb{R}^{m}$ be the argument of $\mathcal{M}$ in (22), and define

$$
\begin{gathered}
\mathcal{N}_{1}\left(\xi_{1}\right):=\left\{(p, x, \lambda) \mid \xi_{1}=\mathcal{L}(p, x, \lambda)\right\} \\
\mathcal{N}_{2}\left(\xi_{2}, \xi_{3}\right):=\left\{(p, x, \lambda) \mid\left(\begin{array}{c}
\xi_{2}+\lambda \\
\xi_{3}+f(x)
\end{array}\right) \in \operatorname{gph} N_{E^{\circ}}\right\} .
\end{gathered}
$$

It follows that $\mathcal{M}(\xi)=\mathcal{N}_{1}\left(\xi_{1}\right) \cap \mathcal{N}_{2}\left(\xi_{2}, \xi_{3}\right)$. By virtue of [17, Theorem 2.5], the assertion holds provided

(i) $\mathcal{N}_{1}$ is calm at $\left(0_{\mathbb{R}^{n}}, \bar{p}, \bar{x}, \bar{\lambda}\right)$ and $\mathcal{N}_{1}^{-1}$ has the Aubin Lipschitz-like property around $\left(\bar{p}, \bar{x}, \bar{\lambda}, 0_{\mathbb{R}^{n}}\right)$

(ii) $\mathcal{N}_{2}$ is calm at $\left(0_{\mathbb{R}^{2 m}}, \bar{p}, \bar{x}, \bar{\lambda}\right)$, and

(iii) multifunction $\xi_{1} \rightrightarrows A\left(\xi_{1}\right):=\mathcal{N}_{2}(0) \cap N_{1}\left(\xi_{1}\right)$ is calm at $\left(0_{\mathbb{R}^{n}}, \bar{p}, \bar{x}, \bar{\lambda}\right)$.

Assumption (i) holds true because of the surjectivity of $\nabla_{p} F(\bar{p}, \bar{x})$. Assumption (ii) follows immediately from the calmness of $\mathcal{N}$. So it suffices to verify assumption (iii). We show that multifunction $A$, in fact, has a stronger Aubin Lipschitz-like property around $\left(0_{\mathbb{R}^{n}}, \bar{p}, \bar{x}, \bar{\lambda}\right)$. To this end, we invoke the coderivative criterion [38, Theorem 9.40]:

$$
\bar{D}^{*} A(0, \bar{p}, \bar{x}, \bar{\lambda})=\{0\} .
$$

In our setting, this criterion provides, by using of standard calculus rules, the sufficient condition

$$
-(\nabla \mathcal{L}(\bar{p}, \bar{x}, \bar{\lambda}))^{T} y \in N_{\mathcal{N}_{2}(0)}(\bar{p}, \bar{x}, \bar{\lambda}) \quad \Rightarrow \quad y=0
$$

guaranteeing the Aubin Lipschitz-like property of $A$ around $\left(0_{\mathbb{R}^{n}}, \bar{p}, \bar{x}, \bar{\lambda}\right)$. Clearly, $\mathcal{N}_{2}(0)=\mathbb{R}^{n} \times \mathcal{N}(0)$. It follows that

$$
N_{\mathcal{N}_{2}(0)}(\bar{p}, \bar{x}, \bar{\lambda})=\left\{0_{\mathbb{R}^{n}}\right\} \times N_{\mathcal{N}(0)}(\bar{x}, \bar{\lambda}),
$$

and consequently implication (24) holds true by virtue of the assumed surjectivity of $\nabla_{p} F(\bar{x}, \bar{p})$. We conclude that all assumptions (i)-(iii) are fulfilled and so the assertion has been established.

Remark 1 For the verification of the calmness of $\mathcal{N}$ at $\left(0_{\mathbb{R}^{2 m}}, \bar{x}, \bar{\lambda}\right)$ we refer to $[13$, Section 3].

Let us comment on the relationship between Theorem 5 and some existing results about stability properties of mappings $S, S^{e}$. In the landmark paper [36], the author considered GE (16) for a general convex set $C$ and derived a sufficient condition for the strong metric regularity of $S^{-1}$ at $(\bar{x}, \bar{p})$. Moreover, he considered also the mapping $S^{e}$ in the case when (16) amounts to the canonically perturbed KKT conditions for a nonlinear program with the constraint set $C$ given by (1). He showed that, in this case, $\left(S^{e}\right)^{-1}$ is strongly metrically regular at the reference triple $(\bar{x}, \bar{\lambda}, \bar{p})$ provided LICQ and the Strong Second Order Sufficient Condition (SSOSC) hold. As proved later in [7], these conditions are not only sufficient but also necessary whenever $\bar{x}$ is a local minimum 
of the considered nonlinear program for the reference value $\bar{p}$. Note that, under strong metric regularity of $\left(S^{e}\right)^{-1}$, the coderivative $\bar{D}^{*} S^{e}(\bar{p}, \bar{x}, \bar{\lambda})$ can be computed by using the standard tools of generalized differential calculus, cf. [29, Proposition 3.2]. In Theorem 5, we provide an upper estimate of $\bar{D}^{*} S^{e}(\bar{p}, \bar{x}, \bar{\lambda})$ under two other conditions the first of which, namely RMFCQ, is substantially weaker than LICQ. Theorem 5 is also related with the corresponding results in $[18,28]$ where upper estimates of $\bar{D}^{*} S(\bar{p}, \bar{x})$ and $\bar{D}^{*} S^{e}(\bar{p}, \bar{x}, \bar{\lambda})$ were computed under MFCQ and appropriate second order qualification conditions.

Our results can be used, e.g., in deriving optimality/stationarity conditions in hierarchical equilibrium problems where GE (16) governs the equilibrium on the lower level or in some other sensitivity/stability issues.

Acknowledgements We acknowledge gratefully the very valuable suggestions of the reviewers which helped us to improve some statements and the overall presentation. The research was partially supported by the Australian Research Council, project DP110102011; Belarus State Research Program "Convergence", section "Mathematical Methods", grant 1.4.3; and the Grant Agency of the Czech Republic, project P402/12/1309.

\section{References}

1. Abadie, J.: On the Kuhn-Tucker theorem. In: Nonlinear Programming (NATO Summer School, Menton, 1964), pp. 19-36. North-Holland, Amsterdam (1967)

2. Andreani, R., Haeser, G., Schuverdt, M.L., Silva, P.: A relaxed constant positive linear dependence constraint qualification and applications. Math. Program., Ser. A 135 255-273 (2012)

3. Andreani, R., Haeser, G., Schuverdt, M.L., Silva, P.: Two new weak constraint qualifications and applications. SIAM J. Optim. 22(3), 1109-1135 (2012)

4. Andreani, R., Martinez, J.M., Schuverdt, M.L.: On the relation between constant positive linear dependence condition and quasinormality constraint qualification. J. Optim. Theory Appl. 125(2), 473-485 (2005)

5. Bazaraa, M.S., Sherali, H.D., Shetty, C.M.: Nonlinear Programming. Theory and Algorithms, third edn. Wiley-Interscience [John Wiley \& Sons], Hoboken, NJ (2006)

6. Bazaraa, M.S., Shetty, C.M.: Foundations of Optimization. Springer-Verlag, Berlin (1976). Lecture Notes in Economics and Mathematical Systems, Vol. 122

7. Dontchev, A.L., Rockafellar, R.T.: Characterizations of strong regularity for variational inequalities over polyhedral convex sets. SIAM J. Optim. 6(4), 1087-1105 (1996)

8. Dontchev, A.L., Rockafellar, R.T.: Implicit Functions and Solution Mappings. A View from Variational Analysis. Springer Monographs in Mathematics. Springer, Dordrecht $\underline{(2009)}$

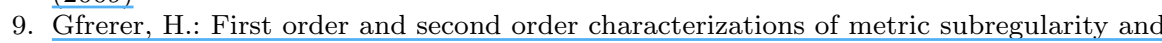
calmness of constraint set mappings. SIAM J. Optim. 21(4), 1439-1474 (2011)

10. Guignard, M.: Generalized Kuhn-Tucker conditions for mathematical programming problems in a Banach space. SIAM J. Control 7, 232-241 (1969)

11. Henrion, R., Jourani, A., Outrata, J.: On the calmness of a class of multifunctions. SIAM J. Optim. 13(2), 603-618 (2002)

12. Henrion, R., Outrata, J.V.: Calmness of constraint systems with applications. Math. Program., Ser. B 104(2-3), 437-464 (2005)

13. Henrion, R., Outrata, J.V., Surowiec, T.: On the co-derivative of normal cone mappings to inequality systems. Nonlinear Anal. 71(3-4), 1213-1226 (2009)

14. Henrion, R., Outrata, J.V., Surowiec, T.: On regular coderivatives in parametric equilibria with non-unique multipliers. Math. Program., Ser. B 136, 111-131 (2012)

15. Ioffe, A.D., Outrata, J.V.: On metric and calmness qualification conditions in subdifferential calculus. Set-Valued Anal. 16(2-3), 199-227 (2008) 
16. Janin, R.: Directional derivative of the marginal function in nonlinear programming. In: A.V. Fiacco (ed.) Sensitivity, Stability and Parametric Analysis, Mathematical Programming Studies, vol. 21, pp. 110-126. Springer Berlin Heidelberg (1984)

17. Klatte, D., Kummer, B.: Nonsmooth Equations in Optimization. Regularity, Calculus, Methods and Applications, Nonconvex Optimization and its Applications, vol. 60. Kluwer Academic Publishers, Dordrecht (2002)

18. Levy, A.B., Mordukhovich, B.S.: Coderivatives in parametric optimization. Math. Program., Ser. A 99(2), 311-327 (2004)

19. Lu, S.: Implications of the constant rank constraint qualification. Math. Program., Ser. A 126(2), 365-392 (2011)

20. Lu, S.: Relation between the constant rank and the relaxed constant rank constraint qualifications. Optimization 61(5), 555-566 (2012)

21. Mangasarian, O.L., Fromovitz, S.: The Fritz John necessary optimality conditions in the presence of equality and inequality constraints. J. Math. Anal. Appl. 17, 37-47 $\underline{(1967)}$

22. Minchenko, L., Stakhovski, S.: On regularity conditions in mathematical programming problems. Dokl. Nats. Akad. Nauk Belarusi 53(5), 26-31, 123 (2009). In Russian

23. Minchenko, L., Stakhovski, S.: About generalizing the Mangasarian-Fromovitz regularity condition. In: Dokl. BGUIR, 8, pp. 104-109 (2010). In Russian

24. Minchenko, L., Stakhovski, S.: On relaxed constant rank regularity condition in mathematical programming. Optimization 60(4), 429-440 (2011)

25. Minchenko, L., Stakhovski, S.: Parametric nonlinear programming problems under the relaxed constant rank condition. SIAM J. Optim. 21(1), 314-332 (2011)

26. Minchenko, L., Tarakanov, A.: On error bounds for quasinormal programs. J. Optim. Theory Appl. 148(3), 571-579 (2011)

27. Mordukhovich, B.S.: Variational Analysis and Generalized Differentiation. I: Basic Theory. Springer-Verlag, Berlin (2006)

28. Mordukhovich, B.S., Outrata, J.V.: Coderivative analysis of quasi-variational inequalities with applications to stability and optimization. SIAM J. Optim. 18(2), 389-412 (2007)

29. Outrata, J.V.: A generalized mathematical program with equilibrium constraints. SIAM J. Contr. Optim. 38, 1623-1638 (2000)

30. Outrata, J.V., Kočvara, M., Zowe, J.: Nonsmooth Approach to Optimization Problems with Equilibrium Constraints, Nonconvex Optimization and Its Applications, vol. 28. Kluwer Academic Publishers, Dordrecht (1998)

31. Ozdaglar, A.E., Bertsekas, D.P.: The relation between pseudonormality and quasiregularity in constrained optimization. Optim. Methods Softw. 19(5), 493-506 (2004)

32. Penot, J.P.: A new constraint qualification condition. J. Optim. Theory Appl. 48(3), 459-468 (1986)

33. Qi, L., Wei, Z.: On the constant positive linear dependence condition and its application to SQP methods. SIAM J. Optim. 10(4), 963-981 (2000)

34. Ralph, D., Dempe, S.: Directional derivatives of the solution of a parametric nonlinear program. Math. Programming, Ser. A 70(2), 159-172 (1995)

35. Robinson, S.M.: Stability theory for systems of inequalities. II. Differentiable nonlinear systems. SIAM J. Numer. Anal. 13(4), 497-513 (1976)

36. Robinson, S.M.: Strongly regular generalized equations. Math. Oper. Res. 5(1), 43-62 (1980)

37. Rockafellar, R.T.: Convex Analysis. Princeton Mathematical Series, No. 28. Princeton University Press, Princeton, N.J. (1970)

38. Rockafellar, R.T., Wets, R.J.B.: Variational Analysis. Springer-Verlag, Berlin (1998) 